of restitution for the coals examined varied from 0.75 to 0.90 , values considerably higher than those got for metals such as copper $(0.62)$ and mild steel $(0 \cdot 73)$.

The coals which exhibited a well-defined minimum in the 'restitution-impact energy' curves were all bright coals which are in general much more brittle and friable than durains and anthracites, and in which occur visible cracks and well-defined cleavage planes. The depth of the minimum and the value of the impact energy at which it occurred varied from coal to coal. The minimum could indicate that at each impact an absorption of energy occurs in addition to the elastic strain energy absorbed. The additional amount of energy absorbed would initially increase from zero at zero impact energy, but rapidly reach some constant value independent of the impact energy of the sphere.

While the mechanism of this additional absorption of energy remains obscure, the effect has been suspected by previous workers. Holland ${ }^{3}$ observed that coals subjected to high compression stresses exhibited hysteresis and permanent set, and only 85 per cent of the energy was released on removing the stress, 15 per cent strain energy being absorbed as permanent strain or appearing as heat. He suggested that the violence of rupture observed indicated that a large portion of this energy was released on failure of the specimen. In their mathematical treatment of coal breakage, Bennett, Brown and Crone ${ }^{5}$ formulated the hypothesis that, under stresses producing breakage, coals behave as brittle materials with a random distribution of inner planes of weakness. On fracture the energy is absorbed in forming new surfaces of the fragmented product, and in the creation of fresh inner weaknesses. As Heywood ${ }^{6}$ pointed out, this hypothesis neglects any elastic stræin energy which may be absorbed, that is, assumes perfect elasticity. The experiments described above show that, with impacts too small to produce fracture, elastic deformations occur which absorb energy (since the coefficient of restitution is less than 1) and, in addition, energy is absorbed by some other mechanism in the case of low-rank bright coals. Most of the specimens used were kindly lent by Dr. Bangham of the British Coal Utilisation Research Association.

$$
\begin{aligned}
& \text { C. G. Cannon. } \\
& \text { W. H. George. }
\end{aligned}
$$

Physics Department,

Chelsea Polytechnic, London, S.W.3.

'Cannon and George, Nature, 150, 690 (1942).

Stopes, Fuel, 14, 4 (1935).

- Holland, Amer. Inst. Min. Met. Eng., Tech. Pub. 1406 (1942) 19 refs.

- Bangham and Maggs, "Ultrafine Structure of Coals and Cokes" (B.C.U.R.A., 1944),

s Bennett, Brown and Crone, J. Inst. Fuel, 10, 22 (1936); 14, 111 (1941).

Heywood (discussion of ref. 5 above), J. S. Wales Inst. Eng. (Octoher 1941).

\section{Explanation of the Broadness and other Characteristics of Association Bands}

THE broadness of the OH, NH or similar bands of pure liquids is well known, and there have been many postulates regarding its origin ${ }^{1,2,3}$. We wish to show here that it is not necessary to assume any involved postulates regarding it, as it can be explained merely from the modern ideas of the mechanism of the formation of the hydrogen bond.
Pauling has shown that a covalent link has a partially ionic character. In the case of $\mathrm{OH}$, for example, $\mathrm{H}$ is sometimes an atom enjoying $\mathbf{a}$ shared pair of electrons and sometimes a mere proton, the pair of electrons being attracted towards $\mathrm{O}$ by its electronegativity. The two states alternate with what is called a resonance frequency. During the time $H$ is a proton, it can approach another negative atom, say, $O$, for intense attractive interaction. Thus the $\mathrm{H}$ atom in a hydrogen bond moves between two points, say $A$ and $B, A$ being the position nearest its parent $O$, and where it rests ordinarily in the absence of a hydrogen bond:

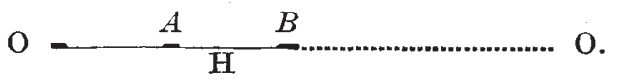

Now, corresponding to each point of occupation by $\mathrm{H}$ on $A B$, there will be an $\mathrm{OH}$ band, with its own frequencies, and as the force constant will go on decreasing on proceeding from $A$ to $B$, the breadth of the band is inherent in the formation of a hydrogen bond. Some other pertinent facts associated with hydragen bonds are also explainable on the above mechanism.

(1) The breadth of the $\mathrm{OH}$ band will be greater the stronger the hydrogen bond. As $A B$ increases with the strength of the hydrogen bond, the breadth increases. Thus carboxylic acids possessing the strongest hydrogen bonds have the widest character of the association bands.

(2) As the concentration of molecules containing $\mathrm{OH}$ increases in an inert liquid, the association band will develop as a whole, that is, the character of the band remains independent of the concentration and only the intensity increases. As the association band due to hydrogen bonds arises in a complete form from each pair of molecules involved in a single hydrogen bond, the fact noted above follows.

(3) Since $A$ is the limit of travel of the $\mathrm{H}$ proton (this being its rest position in the absence of hydrogenbond formation), the high frequency limit of the association band will end sharply at the high-frequency limit of the normal unassociated OH band.

University Physics Department,

R. Parshad.

Physics Laboratory,

Government College,

Lahore.

March 1.

1 Bauer and Magat, J. Phys. et le Radium, 9, 319 (1938).

2 Badger and Bauer, J. Chem. Phys., 5, 839 (1937).

- Sutherland, Trans. Farad. Soc., 36, 889 (1940).

\section{Hæmolytic Disease of the New-born caused by the Antibody St}

RECENTLY hæmolytic disease of the new-born was described $^{1}$ where the $R h+$ mother had in her serum an antibody since designated $S t t^{2}$ or $\gamma^{3}$. From the observations of Race, Taylor and their collaborators $4,5,6$ the reactions of the known allelomorphs of the $R h$ gene with $S t$ serum may be summarized thus :

St serum $\quad \begin{array}{ccccccc}R h_{2} & R h_{0} & R h^{*} & r h & R h_{1} & R h^{\prime} & R h_{2}\end{array}$

An eighth allelomorph, $R h_{y}$, which is $S t^{-}$, is postulated by R. A. Fisher ${ }^{3,6}$. 